\title{
Validation and Diagnostic Usefulness of Gastroesophageal Reflux Disease Questionnaire in a Primary Care Level in Mexico
}

\author{
Miguel Angel Zavala-Gonzáles, ${ }^{1}$ Amyra Ali Azamar-Jacome, ${ }^{1}$ Arturo Meixueiro-Daza, ${ }^{1}$ Antonio Ramos de la Medina, ${ }^{2}$ Job \\ Reyes-Huerta J, ${ }^{1}$ Federico Roesch-Dietlen ${ }^{1}$ and José María Remes-Troche ${ }^{1 *}$ \\ ${ }^{1}$ Laboratory of Digestive Physiology and Gastrointestinal Motility, Instituto de Investigaciones Médico - Biológicas, Universidad Veractuzana, \\ Veractuz, México; and ${ }^{2}$ Regional Hospital of High Specialty of Veracruz, Veractuz, México
}

\begin{abstract}
Background/Aims
Different non-invasive diagnostics strategies have been used to assess patients with gastroesophageal reflux. Gastroesophageal reflux disease (GERD) questionnaire (GerdQ) is a 6-item, easy to use questionnaire that was developed primarily as a diagnostic tool for GERD in primary care. Our aim was to validate and assess diagnostic utility of GerdQ questionnaire in Mexican patients in the primary care setting.
\end{abstract}

\section{Methods}

The study was performed in 3 phases: (1) a questionnaire translation and comprehension study $(n=20)$, (2) are a reproducibility and validation study (50 patients and 50 controls) and (3) a study to assess the clinical utility in 252 subjects with GERD symptoms. Diagnostic accuracy was calculated using endoscopy and/or pH-metry as the gold standard.

\section{Results}

Internal consistency measured by the Cronbach's a coefficient was 0.81 for patients and 0.90 for healthy controls, with a mixed coefficient of 0.93 . Reproducibility for GerdQ was very good and its discriminating validity was $88 \%$. Most of the patients with erosive reflux and non-erosive reflux with abnormal pH-metry had scores $>8$, meanwhile most of the patients with functional heartburn and hypersensitive esophagus had $<8$. Sensitivity, specificity and positive predictive value of GerdQ compared to the gold standard were $72 \%, 72 \%$ and $87 \%$, respectively.

Received: February 13, 2014 Revised: June 11, 2014 Accepted: June 19, 2014

(c) This is an Open Access article distributed under the terms of the Creative Commons Attribution Non-Commercial License (http://creativecommons. org/licenses/by-nc/3.0) which permits unrestricted non-commercial use, distribution, and reproduction in any medium, provided the original work is properly cited.

*Correspondence: José María Remes-Troche, MD

Laboratory of Digestive Physiology and Gastrointestinal Motility, Instituto de Investigaciones Médico - Biológicas, Universidad Veracruzana, Veracruz. Iturbide SN, Colonia Flores Magón, CP 91400, Veracruz, México

Tel: +52-229-922-3292, Fax: +52-229-202-1231, E-mail: joremes@uv.mx

Financial support: This study was funded in part by Astra Zeneca.

Conflicts of interest: José María Remes-Troche has served as a speaker, a consultant and an advisory board member for Astra Zeneca, Takeda Pharmaceuticals, Janssen, Asofarma, Almirall, Alfa-Wasserman and BMS. Also, José María Remes-Troche has received research funding from Astra Zeneca and Asofarma. Arturo Meixueiro-Daza has served as a speaker for Takeda Pharmaceuticals and Asofarma. Antonio Ramos de la Medina has served as a speaker, a consultant and an advisory board member for Takeda Pharmaceuticals. Miguel Angel Zavala-Gonzáles, Amyra Ali Azamar-Jacome, Job Reyes-Huerta, and Federico Roesch-Dietlen do not have any potential conflicts (financial, professional or personal) that are relevant to this manuscript.

Author contributions: Miguel Angel Zavala-Gonzáles, Amya Ali Azamar-Jacome, Job Reyes-Huerta, Antonio Ramos de la Medina, Federico Roesch-Dietlen and Jose María Remes-Troche performed the research; Miguel Angel Zavala-Gonzáles and Amyra Ali Azamar-Jacome collected and analyzed the data; José María Remes-Troche designed the research study and wrote the paper; Antonio Ramos de la Medina and Federico Roesch contributed to the design of the study. All authors approved the final version of the article, including the authorship list.

ORCID: José María Remes-Troche, http://orcid.org/0000-0001-8478-9659. 


\section{Conclusions}

In Mexico, the GerdQ questionnaire Spanish validated version is useful for GERD diagnosis in the primary care setting.

(J Neurogastroenterol Motil 2014;20:475-482)

Key Words

Gastroesophageal reflux; Mexico; Questionnaires; Sensitivity and specificity

\section{Introduction}

In the last decades, important diagnostics and therapeutics progress has been achieved, which has allowed a better knowledge of physiopathology of the gastroesophageal reflux disease (GERD). ${ }^{1}$ Currently, GERD is considered as a heterogeneous multi-symptomatic disease, thus, it is reasonable to say that there is no gold standard test. Usually, a combination of several diagnostic tools (questionnaires, endoscopy, etc) is used to make the diagnosis. ${ }^{2}$ The decision about which test will be used, when it will be used and what will be the benefit for diagnosis depends on several factors, such as costs and avaibility at the primary care level.

Several strategies have been developed to diagnose GERD without the need of invasive studies. Questionnaires are one of these tools, which allow an objective assessment of symptoms. ${ }^{3}$ These questionnaires are constructs that usually include a series of questions to assess severity (most of the times using scales like Likert's scale), frequency, related phenomena, nighttime symptoms and sometimes quality of life. Advantages of questionnaires include that they can be self-administered, used as screening tools, their low cost and can be applied to any subject suffering from GERD.

In the literature, there are at least 20 questionnaires described; Carlsson-Dent, ${ }^{4}$ ReQuest ${ }^{5}$ and GerdQ $^{6}$ questionnaires are the most used. Once a questionnaire is completed a score is established and there is a pre-established cut off point, which allow determining likelihood of GERD. For instance, a score higher than 8 (scale from 0 to 18 ) in the GerdQ is related to a likelihood of esophageal erosions or abnormal $\mathrm{pH}$-metry in over $50 \%$ of the cases. ${ }^{6}$

The GerdQ is a 6-item tool developed to contribute to GERD diagnosis based on the symptoms found in patients who attend at primary care. One advantage of this questionnaire is that it was developed from 3 questionnaires that assess different aspects of GERD: the questionnaire for reflux disease (reflux disease questionnaire), ${ }^{7}$ the scale for assessment of gastrointestinal symptoms (gastrointestinal symptom rating scale) ${ }^{8}$ and the reflux disease impact scale (gastroesophageal reflux disease impact scale). ${ }^{9}$ Thus, GerdQ is useful and helps to made diagnostic decisions since it can differentiate between symptoms due to temporary reflux and frequent symptoms (which affect individual's daily performance). Also, GerdQ permits monitoring the effects of treatments on long-term patient's symptoms.

In this study, our aim was to validate and assess diagnostic usefulness of GerdQ questionnaire for the diagnostic of GERD in Mexican patients who seek for first level medical care (general practice).

\section{Materials and Methods}

The study was performed in 3 phases: (1) a questionnaire translation and comprehension study $(\mathrm{n}=20),(2)$ a reproducibility and validation study ( 50 patients and 50 controls) and (3) a study to assess the clinical utility in 252 subjects with GERD symptoms.

\section{Translation and Comprehension Study}

\section{Questionnaire}

The questionnaire is composed of 6 items, 4 of which assess symptoms and situations considered positive predictors for GERD diagnosis: heartburn, regurgitations, disorders related to sleep and use of over the counter products. Other 2 items assess 2 symptoms considered negative predictors for reflux, such as nausea and epigastric pain. Patient answers each question about symptoms frequency during last week using a Likert like scale from 0 to 3 for positive predictors and from 3 to 0 for negative predictors (Table 1). The maximum score that can be obtained is 18 .

\section{Translation}

Two independent Gastroenterologists natives of Mexico (ARD and JMRT) performed translation of the English text into Spanish. Each investigator's translations were compiled to pro- 
Table 1. Gastroesophageal Reflux Disease Questionnaire

\begin{tabular}{|c|c|c|c|c|}
\hline \multirow{2}{*}{ Questions } & \multicolumn{4}{|c|}{ Frequency score for symptoms (points) } \\
\hline & 0 day & 1 day & 2-3 days & 4-7 days \\
\hline How often did you have a burning feeling behind your breastbone (heartburn)? & 0 & 1 & 2 & 3 \\
\hline $\begin{array}{l}\text { How often did you have stomach contents (liquid or food) } \\
\text { moving upwards to your throat or mouth (regurgitation)? }\end{array}$ & 0 & 1 & 2 & 3 \\
\hline How often did you have pain in the center of the upper stomach? & 3 & 2 & 1 & 0 \\
\hline How often did you have nausea? & 3 & 2 & 1 & 0 \\
\hline $\begin{array}{l}\text { How often did you have difficulty getting a good night's sleep because of } \\
\text { your heartburn and/or regurgitation? }\end{array}$ & 0 & 1 & 2 & 3 \\
\hline $\begin{array}{l}\text { How often did you take additional medication for your heartburn and/or regurgitation, } \\
\text { other than what the physician told you to take (such as Tums, Rolaids and Maalox)? }\end{array}$ & 0 & 1 & 2 & 3 \\
\hline
\end{tabular}

duce finally the first draft of agreed joint translation.

\section{Questionnaire comprehension}

The questionnaire's first draft was applied by means of clinical interview to a small sample of patients with a previous symptomatic and endoscopic diagnosis of GERD $(n=20)$, whose education level was low (elementary education) to ensure understanding by any type of patients. The validation process was performed in the Laboratory of Digestive Physiology and Gastrointestinal Motility, Universidad Veracruzana. According to the responses, questions and answers were discussed with patients and appropriate changes were made afterwards.

\section{Questionnaire back-translation into English}

A native translator not familiar with the original English version performed the back-translation of the Spanish version. Afterwards a team composed by the investigators and the translator compared the back-translation to the original one for review possible discrepancies and made proper changes.

\section{Validation and Reproducibility}

For questionnaire validation, 50 patients ( $60 \%$ women, 42.3 mean age) with GERD (endoscopic or by abnormal ambulatory 24-hour $\mathrm{pH}[<4 \%,>4.2 \%]$ ) and 50 controls (matched by age and gender) were assessed. Patients were older than 18 years of age and had symptoms suggestive of GERD in the past 3 months. Patients presenting with alarm symptoms such as unintentional weight loss, severe or progressive dysphagia or gastrointestinal (GI) bleeding were excluded from participation. Controls were selected from a gastric cancer-screening program where asymptomatic subjects undergo upper GI endoscopy. All subjects completed questionnaires in 2 stages: assessment (1) pri- or to the endoscopy study, and assessment (2) between 7 and 15 days following the first evaluation, (usually when they attended for follow-up consultation). Between assessment 1 and 2 patients were instructed to do not use any medication.

Internal consistency was calculated using the Cronbach's $\alpha$ coefficient value for all six items. GERD diagnosis was considered positive if questionnaire score was $>8$. Reproducibility was measured by means of test-retest comparing answers from assessment 1 with answers from assessment 2 (McNemar's test). Discriminating validity between patients and controls was assessed by means of Pearson's $\chi^{2}$ test. Content's validity, although it is not a statistical test, it is a wise analysis performed by specialists in the field about representation and relevance of proposed items for GerdQ. This analysis was conducted by 2 experts in the field (ARM and JMRT).

\section{Gastroesophageal Reflux Disease Questionnaire's Clinical Usefulness}

\section{Patients}

Three hundred consecutive patients with symptoms of reflux (heartburn or regurgitations) whom attended to 3 primary care centers were invited to participate in this study during the period between January 2011 and October 2012. Patients were older than 18 years of age and had symptoms suggestive of GERD in the past 3 months. Patients presenting with alarm symptoms such as unintentional weight loss, severe or progressive dysphagia or GI bleeding were excluded from participation.

\section{Study protocol}

All subjects were referred for assessment at the Laboratory of Digestive Physiology and Gastrointestinal Motility, Universidad 
Veracruzana, Veracruz, Mexico. Once the informed consent form was signed and voluntary acceptance from subjects was granted, the following study protocol was followed.

Gastroesophageal reflux disease questionnaire. All subjects completed the Spanish validated GerdQ questionnaire at the baseline visit. ${ }^{6}$ GerdQ was considered positive if the score was $>8$. Two out of the 6 items ( 5 and 6 ) assessed GERD impact on quality of life, and a score $>3$ in this subscale was related with higher impact of disease on subject's health. All questionnaires were administered and assessed by 2-blinded investigators (AAAJ and MAZG).

Upper gastrointestinal endoscopy. All patients underwent conventional upper GI endoscopy (GIF-160, Olympus endoscopy, Tokyo, Japan) with sedation. Patients were classified as having erosive GERD when esophageal erosions were found, and the Los Angeles classification was used. ${ }^{10}$ Endoscopists were blinded to results of the surveys previously administered to the patients.

Esophageal pH-metry. When patients had normal endoscopy, then they were scheduled for an ambulatory 24-hour esophageal pH-metry. All patients withdraw medications such as proton pump inhibitors, histamine $\mathrm{H} 2$ receptors blockers and antacids 7 days prior their procedure. After an overnight fasting, a $\mathrm{pH}$ catheter (previously calibrated with standard solutions at $\mathrm{pH}$ 1 and 7) was introduced through the nostrils and the tip of the probe was located $5 \mathrm{~cm}$ above the lower esophageal sphincter previously located with esophageal manometry. Once it has been introduced, the catheter was plugged in a portable digital recorder (Sandhill, Denver, Colorado, USA) and patients were instructed to record time of his/her symptoms (heartburn), fed and supine position. Patients were advised to keep their regular diet and to perform regular activities during the study. $\mathrm{pH}$-metry was considered abnormal when percentage of total time with $\mathrm{pH}<4$ was higher than $4.2 \%{ }^{11}$ Symptoms index (SI) was calculated as the percentage of symptoms which occurred during acid reflux episodes $(\mathrm{pH}<4)$; it was considered positive if symptoms-reflux episodes correlation was $>50 \%$. $^{12}$ According to $\mathrm{pH}$-metry, patients were classified in 3 groups: (1) patients with abnormal esophageal exposure to acid ( $\% \mathrm{pH}<4,>4.2 \%$ ), (2) patients with normal esophageal exposure to acid but SI positive (also called hypersensitive esophagus) and (3) patients with normal esophageal exposure to acid with SI negative (patients classified as functional heartburn.)

\section{Statistical Methods}

Absolute and relative frequencies were used to describe nominal variables. According to its distribution, we used mean and standard deviation, or median and ranges for continue variables. Considering that GerdQ questionnaire was positive when score was $>8$, diagnostic accuracy, sensitivity, specificity and predictive values were calculated taking into consideration as "as gold standards" the prior clinical diagnosis (made by a general practitioner), an abnormal endoscopy, an abnormal 24-hour $\mathrm{pH}$ metry and combination of endoscopy and 24-hour pH-metry results. Comparison between proportions was performed using the $\chi^{2}$ test, $U$ of Mann-Whitney and Kruskal-Wallis when appropriate. Also, a multinomial logistic regression was performed. Statistical significance was established when $P$ was $<0.05$.

\section{Results}

\section{Validation and Reproducibility}

Internal consistency measured by the Cronbach's coefficient for the 6 items completed was of 0.81 for patients and 0.90 for healthy controls, with a combined coefficient of 0.93 . Reproducibility for GERD diagnosis between assessment 1 and 2 is shown in Table 2. As shown in Table 2, $86 \%$ of subjects had a score $>8$ in assessment 1 and $88 \%$ in assessment 2. McNemar's test showed that mismatch occurred was by chance $(P=1.000)$.

Regarding discriminating validity, as shown in Table 3, in the baseline assessment (1) the questionnaire showed that only 2 (4\%) of control subjects had a score $>8$ compared to 43 (86\%) of patients with GERD $(P=0.000)$. Mean GerdQ value was 4 in controls and 11 in GERD patients $(P=0.001)$.

Table 2. Patients' Frequencies With a Gastroesophageal Reflux Disease Diagnosis Based on Gastroesophageal Reflux Disease Questionnaire $>8$ at Assessment 1 and at Assessment 2 (Reproducibility Study)

\begin{tabular}{lllcc}
\hline & & \multicolumn{3}{c}{ GERD diagnosis (GerdQ $>$ 8) } \\
& & \multicolumn{3}{c}{ Assessment 2 } \\
\cline { 3 - 5 } & & No & Yes & Total \\
\hline GERD diagnosis & No & $4(8)$ & $3(6)$ & $7(14)$ \\
$($ GerdQ $>$ 8) & Yes & $2(4)$ & $41(82)$ & $43(86)$ \\
Assessment 1 (n [\%]) & Total & $6(12)$ & $44(88)$ & $50(100)$ \\
\hline
\end{tabular}

GERD, gastroesophageal reflux disease; GerdQ, gastroesophageal reflux disease questionnaire. 
Regarding the content analysis, the 2 experts agreed that questionnaire is an appropriate assessment GERD diagnosis, and questions were clear and adequate.

\section{Clinical Usefulness Assessment}

From a total of 300 assessed subjects who were invited to participate in the study, 252 (84\%) completed the study protocol, and data was analyzed. There were 159 women (73\%). Overall, the mean age and the mean BMI were 49.49 years (range from 18 to 92 ) and $26.5 \pm 6.44 \mathrm{~kg} / \mathrm{m}^{2}$, respectively.

According to endoscopy findings, erosive GERD was found in 110 cases (44\%) and 142 (56\%) had non-erosive GERD (NERD). Seventy (49\%) out of 142 patients with NERD had abnormal exposure to acid. Twenty (28\%) patients out of 72 (51\%) with negative $\mathrm{pH}$-metry, were classified as "hypersensitive esophagus" and 52 (72\%) with functional heartburn (Fig. 1). Baseline characteristics among GERD groups are shown in Table 4.
Overall, mean GerdQ score was $8 \pm 3$ points. Eleven subjects (4\%) had scores from 0 to 2, 121 (48\%) had score from 3 to 7, 54 (22\%) from 8 to 11 and 66 (26\%) higher than 11. Most of the patients with erosive GERD and NERD with abnormal $\mathrm{pH}$ -

Table 3. Patients and control's Percentages With a Diagnosis of Gastroesophageal Reflux Disease Based on Gastroesophageal Reflux Disease Questionnaire $>8$ in Assessment 1 (Discriminating Validation Study)

\begin{tabular}{lccr}
\hline & \multicolumn{3}{c}{$\begin{array}{c}\text { GERD diagnosis (GerdQ }>8) \\
\text { Baseline Assessment }\end{array}$} \\
\cline { 2 - 4 } & No & Yes & Total \\
\hline Patients with GERD (n [\%]) & $7(14)$ & $43(86)$ & $50(100)$ \\
Controls (n [\%]) & $48(96)$ & $2(4)$ & $50(100)$ \\
Total (n [\%]) & $55(55)$ & $45(45)$ & $100(100)$
\end{tabular}

GERD, gastro esophageal reflux disease; GerdQ, gastro esophageal reflux disease questionnaire.
Heartburn + abnormal endoscopy Heartburn + normal endoscopy

Erosive GERD
$(n=110)$
$44 \%$
$\begin{gathered}\text { Abnormal acid } \\ \text { exposure } \\ (n=70) \\ 49 \%\end{gathered}$

Non-erosive GERD

$$
(n=142)
$$$$
56 \%
$$

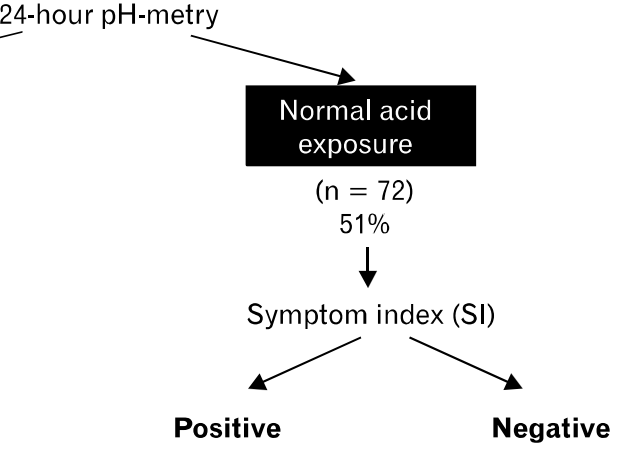

Esophageal hypersensitivity $(n=20)$
$28 \%$
Functional heartbum $(n=52)$ $72 \%$
Figure 1. Patients' distribution according to endoscopic findings and ambulatory 24-hour pH-metry. GERD, gastroesophageal reflux disease.

Table 4. Baseline Characteristics Among Gastroesophageal Reflux Disease Subgroups

\begin{tabular}{lcccc}
\hline & $\begin{array}{c}\text { Erosive GERD } \\
(\mathrm{n}=110)\end{array}$ & $\begin{array}{c}\text { NERD Abnormal } \mathrm{pH} \\
(\mathrm{n}=70)\end{array}$ & $\begin{array}{c}\text { Hypersensitive esophagus } \\
(\mathrm{n}=20)\end{array}$ & $\begin{array}{c}\text { Functional Heartburn } \\
(\mathrm{n}=52)\end{array}$ \\
\hline Gender & & & & $18(34)$ \\
$\quad$ Male (n [\%]) & $59(54)$ & $28(40)$ & $8(40)$ & $34(65)$ \\
$\quad$ Female (n [\%]) & $51(46)$ & $42(60)$ & $12(60)$ & $38 \pm 13$ \\
Age (mean \pm SD, yr) & $42 \pm 17$ & $37 \pm 15$ & $38 \pm 8$ & $24.2 \pm 4.0$ \\
Body mass index (mean $\pm \mathrm{SD})$ & $26.1 \pm 4.2$ & $25.7 \pm 3.0$ & $23.8 \pm 3.0$ & 4.0
\end{tabular}

GERD, gastroesophageal reflux disease; NERD, non-erosive esophageal reflux disease. 
metry had scores $>8$, meanwhile most of the patients with functional heartburn and hypersensitive esophagus had scores $<8$ (Fig. 2).

Diagnostic accuracy, sensitivity, specificity, positive predictive values and negative predictive values for GerdQ compared with other diagnostics tests are shown in Table 5.

One hundred and one patients ( $40 \%)$ had a score equal to or higher than 3 in the disease impact scale, 71 women and 30 male $(P=0.047)$. There were no age differences among patients whose score was $\geq 3$ or $<3$ points in the impact scale (47.6 vs. 50.6 years, $P=0.175)$. Body mass index was similar in both groups (27 vs. $26.02 \mathrm{~kg} / \mathrm{m}^{2}, P=0.438$ ). Patients with erosive GERD were likely to have a score $>3$ in the impact sub-scale compared with patients with NERD (68\% vs. $32 \%, P=0.030$ ). When a multinomial logistic regression was performed the only factor associated to the worst disease impact scale was the presence of erosive GERD $(P=0.003)$.

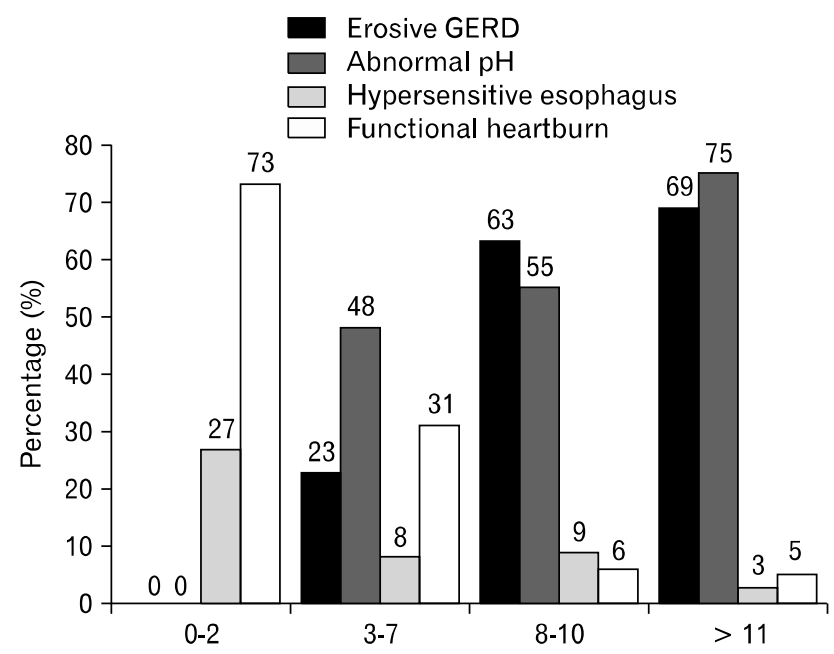

Figure 2. Patients' distribution according to gastroesophageal reflux disease (GERD) phenotype and different gastroesophageal reflux disease questionnaire cut points.

\section{Discussion}

In this study, we showed the results for the formal validation process for the GerdQ questionnaire in the Mexican population. GerdQ is an easy to use questionnaire that has been previously in Europe, USA and China. ${ }^{6,13}$ According to our results, Spanish validated version of GerdQ questionnaire was adequate and with excellent clinimetric properties such a very good consistency, reproducibility and discriminating capacity between healthy subjects and patients. These characteristics are essential to consider that a questionnaire can be a useful diagnostic tool.

Once the questionnaire was validated, we assess clinical usefulness of GerdQ in Mexican patients who sought first level care. Our results showed that their positive predictive value compared to the gold standard (endoscopy and positive $\mathrm{pH}$-metry) was of $86 \%$, which is considered to be very good. In general, it is important to have simple but useful diagnostic tools such as symptom questionnaires in the primary care level. Usually, general practitioners have the challenge to establish a diagnosis with limited resources, but a easy and prompt diagnosis allows to start treatment as soon as possible.

Although there are several diagnostic questionnaires, ${ }^{3}$ GerdQ had some advantages including its simplicity, it allows to assess severity and frequency of symptoms, and have a disease's impact scale (quality of life).

Our findings are very similar to those reported in validations and baseline evaluations of GerdQ in other populations. ${ }^{6}$ For example, in the initial study where the questionnaire was developed ( $\mathrm{n}=308$ subjects), a score $>8$ showed sensitivity and specificity of $64.6 \%$ and $71.4 \%$, respectively, when compared with other clinical diagnosis tools such as the diagnosis made by the physician (gastroenterologists or general practitioner). In our study, sensitivity and specificity compared with former clinical diagnosis was of $54 \%$ and $72 \%$, respectively. More recently, Jonasson et $\mathrm{al}^{14}{ }^{14}$ in the validation study for questionnaire in 169 patients with

Table 5. Diagnostic Accuracy of Gastroesophageal Reflux Disease Questionnaire Compared to Other Diagnostic Tools

\begin{tabular}{lcccccc}
\hline & Prevalence & Sensitivity & Specificity & PPV & NPV & Accuracy \\
\hline GerdQ vs. Endoscopy & 43.65 & 74.55 & 73.24 & 68.33 & 78.79 & 73.81 \\
GerdQ vs. pH-metry & 49.30 & 67.14 & 72.22 & 70.15 & 69.33 & 69.72 \\
GerdQ vs. Endoscopy + pH-metry & 71.43 & 71.67 & 72.22 & 86.58 & 50.49 & 71.83 \\
Prior diagnosis vs. Endoscopy & 43.65 & 53.64 & 71.83 & 59.60 & 66.67 & 63.89 \\
\hline
\end{tabular}

PPV, positive predictive value; NPV, negative predictive value; GerdQ, gastroesophageal reflux disease questionnaire.

Data are presented as \%. 
GERD (93\% erosive) found higher sensitivity (78\%) with lower specificity (50\%). This difference can be explained since in this study, authors were highly biased by referrals and prevalence of erosive GERD. Another explanation for this finding is that in the Norwegian health care system, it is mandatory to objectively check patients suffering from GERD (by means of endoscopy and/or pH-metry) in order to prescribe a proton pump inhibitor; therefore, many of the Jonasson study's patients certainly stopped their over the counter medication prior to this assessment. Therefore, our study compared to that of Jonasson, seems to have a representative sample of things happening at the primary care level where only about half of the patients have erosive GERD. However, in spite of differences that might exist, in our study as in the Jonasson's study, positive predictive values are very high $(86.0 \%$ and $89.1 \%$ ) when compared to the gold standards.

It is worth to emphasize that this questionnaire has been developed for routine use by general practitioner and its results are controversial when used in other populations. For instance, Lacy et $\mathrm{al}^{15}$ assessed GerdQ diagnostic efficacy against 48-hour $\mathrm{pH}$ Bravo system, and showed this questionnaire was not enough to establish the GERD diagnosis. In another study conducted in our country, in a third level site in Mexico City ${ }^{16}$ in 65 patients (mean age of 43 years) compared GerdQ questionnaire with $\mathrm{pH}$-metry and 24-h impedance, sensitivity and specificity were shown to be limited by high prevalence of subjects with diagnosis of functional heartburn and by lack of endoscopic assessment. Both studies are biased regarding referral, since they were conducted in 3rd level sites where there is a high prevalence of refractory GERD and atypical onset; therefore, these samples are not representative of the population, which this GerdQ questionnaire is intended. ${ }^{17}$

Regarding other frequently used questionnaires, in Mexico ReQuest and Carlsson-Dent have been used. In the study where Carlsson-Dent questionnaire was assessed for the diagnosis of GERD, it was administered to 86 patients with NERD and to 39 patients with erosive GERD. It was shown that compared with the 24-hour pH-metry and endoscopy, a score $>4$ in the questionnaire has a sensitivity of $88 \%$ and $94 \%$, respectively. ${ }^{18}$ This study was conducted in a third level of referral, and with a sample size much less assessed than in our study. Concerning studies conducted with ReQuest in Mexico, this tool has been used to measure symptoms intensity before and following dosing of pantoprazole magnesium; however, its diagnostic efficacy is unknown. ${ }^{19}$

Interestingly, our study compared to other studies explored the diagnostics usefulness of GerdQ considering the different phenotypes of GERD and establishing thus, that most of the patients with erosive GERD and NERD with abnormal pHMetry had scores $>8$, meanwhile most of the patients with functional heartburn and hypersensitive esophagus had scores $<8$. That means there seems to be a relation indicating that the high score and higher likelihood of a more aggressive disease; meanwhile on the other hand patients with lower scores but with recurrent symptoms might belong to the spectrum of functional patients with GERD. Although the usefulness of GerdQ questionnaire among GERD subgroups is novel; it may be difficult to assure that erosive GERD is a more aggressive disease than NERD. Recently, NERD is considered a distinct entity with differences in epidemiology, pathophysiology, symptoms and therapeutic response. ${ }^{12}$

Although it is difficult to explain differences among subgroups, it is possible that patients with a more functional presentation of GERD (hypersensitive esophagus and functional heartburn) also have more overlapping with other functional disorders such as functional dyspepsia. Thus, the presence of more severe dyspeptic symptoms is a negative predictor for GERD that may explain lower scores in the GerdQ questionnaire.

Finally, it is important to mention that although GerdQ represents a useful diagnostic tool, it should not be considered as the unique diagnostic test. It can be used as a baseline and/or complementary test if there are no alarm signs. Also, it can be used for monitoring the therapeutic effect of GERD treatments. In those patients with mild symptoms and response to proton pump inhibitors, no other test will be required; however, in case of lack of response, it would be the most appropriate to confirm GERD diagnosis, by means of $\mathrm{pH}$-metry and/or endoscopy, as appropriate.

In Mexico, the GerdQ questionnaire Spanish validated version is useful for gastroesophageal reflux disease diagnosis in the primary care setting.

\section{Acknowledgement}

Mexico Writing support was provided by María del Carmen Rodríguez Luna, Professional Translator.

\section{References}

1. Vakil N, van Zanten SV, Kahrilas P, Dent J, Jones R; Global Consensus Group. The Montreal definition and classification of gastroesophageal reflux disease: a global evidence-based consensus. Am J Gastroenterol 2006;101:1900-1920.

2. Katz PO, Gerson LB, Vela MF. Guidelines for the diagnosis and 
management of gastroesophageal reflux disease. Am J Gastroenterol 2013;108:308-328.

3. Mouli VP, Ahuja V. Questionnaire based gastroesophageal reflux disease (GERD) assessment scales. Indian J Gastroenterol 2011;30: 108-117.

4. Carlsson R, Dent J, Bolling-Sternevald E, et al. The usefulness of a structured questionnaire in the assessment of symptomatic gastroesophageal reflux disease. Scand J Gastroenterol 1998;33:1023-1029.

5. Bardhan KD, Stanghellini V, Armstrong D, Berghöfer P, Gatz G, Mönnikes $\mathrm{H}$. Evaluation of GERD symptoms during therapy. Part I. Development of the new GERD questionnaire ReQuest TM. Digestion 2004;69:229-237.

6. Jones R, Junghard O, Dent J, et al. Development of the GerdQ, a tool for the diagnosis and management of gastro-oesophageal reflux disease in primary care. Aliment Pharmacol Ther 2009;30:1030-1038.

7. Shaw M, Dent J, Beebe T, et al. The Reflux Disease Questionnaire: a measure for treatment response in clinical trials. Health Qual Life Outcomes 2008;6:31.

8. Revicki D, Wood M, Wiklund I, Crawley J. Reliability and validity of the Gastrointestinal Symptom Rating Scale in patients with gastro-oesophageal reflux disease. Qual Life Res 1998;7:75-83.

9. Jones R, Coyne K, Wiklund I. The gastro-oesophageal disease impact scale: a patient management tool for primary care. Aliment Pharm Ther 2007;25:1451-1459.

10. Lundell LR, Dent J, Bennett JR, et al. Endoscopic assessment of oesophagitis: clinical and functional correlates and further validation of the Los Angeles classification. Gut 1999;45:172-180.

11. DeMeester TR, Wang CI, Wernly JA, et al. Technique, indications and clinical use of 24-hour esophageal $\mathrm{pH}$ monitoring. J Thorac Cardiovasc Surg 1980;79:656-670.
12. Fass R, Fennerty MB, Vakil N. Nonerosive reflux disease-current concepts and dilemmas. Am J Gastroenterol 2001;96:303-314.

13. Wong WM, Lam KF, Lai KC, et al. A validated symptoms questionnaire (Chinese GERDQ) for the diagnosis of gastro-oesophageal reflux disease in the Chinese population. Aliment Pharmacol Ther 2003;17:1407-1413.

14. Jonasson C, Wernersson B, Hoff DA, Hatlebakk JG. Validation of the GerdQ questionnaire for the diagnosis of gastro-oesophageal reflux disease. Aliment Pharmacol Ther 2013;37:564-572.

15. Lacy BE, Chehade R, Crowell MD. A prospective study to compare a symptom-based reflux disease questionnaire to 48 -h wireless ph monitoring for the identification of gastroesophageal reflux. Am J Gastroenterol 2011;106:1604-1611.

16. Zavala-Solares M, Carmona-Sanchez R, Saleme Cruz EN, VargasVorackova F, Valdovinos-Diaz MA. Rendimiento del GerdQ en la detección de la enfermedad por reflujo gastroesofágico (ERGE) en una muestra de pacientes mexicanos en centros de tercer nivel de atención. Rev Gastroenterol Mex 2011;76(suppl 2):39.

17. Vakil N, Kahrilas PJ. GERD Diagnosis: pretest probability and the "gold" standard alter outcome. Am J Gastroenterol 2012;107:322323

18. Gómez-Escudero O, Remes-Troche JM, Ruíz JC, Peláez-Luna M, Schmulson MJ, Valdovinos Díaz MA. Diagnostic usefulness of the Carlsson-Dent questionnaire in gastroesophageal reflux disease (GERD). Rev Gastroenterol Mex 2004;69:16-23.

19. López-Alvarenga JC, Orr W, Vargas-Romero JA, et al. Relief of night-time symptoms associated with gastroesophageal reflux disease following 4 weeks of treatment with pantoprazole magnesium: the mexicangastroesophageal reflux disease working group. J Neurogastroenterol Motil 2014;20:64-73. 